\title{
Coorbital Periodic Orbits in the Three Body Problem*
}

\author{
Josep M. Cors ${ }^{\dagger}$ and Glen R. Hall ${ }^{\ddagger}$
}

\begin{abstract}
We consider the dynamics of coorbital motion of two small moons about a large planet which have nearly circular orbits with almost equal radii. These moons avoid collision because they switch orbits during each close encounter. We approach the problem as a perturbation of decoupled Kepler problems as in Poincaré's periodic orbits of the first kind. The perturbation is large but only in a small region in the phase space. We discuss the relationship required among the small quantities (radial separation, mass, and minimum angular separation). Persistence of the orbits is discussed.
\end{abstract}

Key words. coorbital motion, periodic orbits of the first kind, three body problem

AMS subject classifications. 70F $15,70 \mathrm{~F} 07$

PII. S1111111102411304

1. Introduction. In 1981, the Voyager 1 space craft explored and photographed the neighborhood of Saturn (see Aksnes [1]). These photos captured two remarkable small moons subsequently named Janus and Epimetheus. The moons are remarkable because they are of nearly equal mass, and their orbits have nearly equal radii. Because their orbits are so close, their motion is called "coorbital." As implied by Kepler's laws, the inner moon has a smaller period and hence catches up to the outer moon. The moons avoid collision because their mutual gravitation causes them to switch orbits when they approach each other. Figure 1.1 shows a schematic drawing of the paths of the coorbital moons. Figure 1.2 shows computed orbits of the two moons in rotating coordinates. The speed of rotation of the coordinates is that of the orbit of average radius. In this paper, we investigate the dynamics of the motion of a pair of small, comparable mass, coorbital moons as solutions of the Newtonian three body problem.

Several authors have studied the dynamics of coorbital satellites in general and Janus and Epimetheus in particular. One approach is to treat one of the moons as a test particle of zero mass and think of the dynamics as that of the restricted three body problem. Coorbital motion in this situation is very interesting and has been extensively studied both analytically and numerically (see, for example, Salo and Yoder [12] and Llibre and Ollé [4]).

Since the small moons are of comparable mass, it is more natural to treat them equally in the analysis. Two groups have approached the problem from this point of view. One approach is to do a "matching" of two separate dynamics problems - an "outer" problem, where the small moons do not interact, and an "inner" problem, where the interactions dominate the

\footnotetext{
${ }^{*}$ Received by the editors July 18, 2002; accepted for publication (in revised form) by J. Meiss January 20, 2003; published electronically May 23, 2003.

http://www.siam.org/journals/siads/2-2/41130.html

${ }^{\dagger}$ Departament de Matemàtica Aplicada III, Universitat Politècnica de Catalunya, 08240, Manresa, Spain (cors@eupm.upc.es). The work of this author was partially supported by DGICYT grant PB96-1153 and MEC grant PR97-39332370.

${ }^{\ddagger}$ Department of Mathematics and Statistics, Boston University, Boston, MA 02215 (rockford@math.bu.edu).
} 


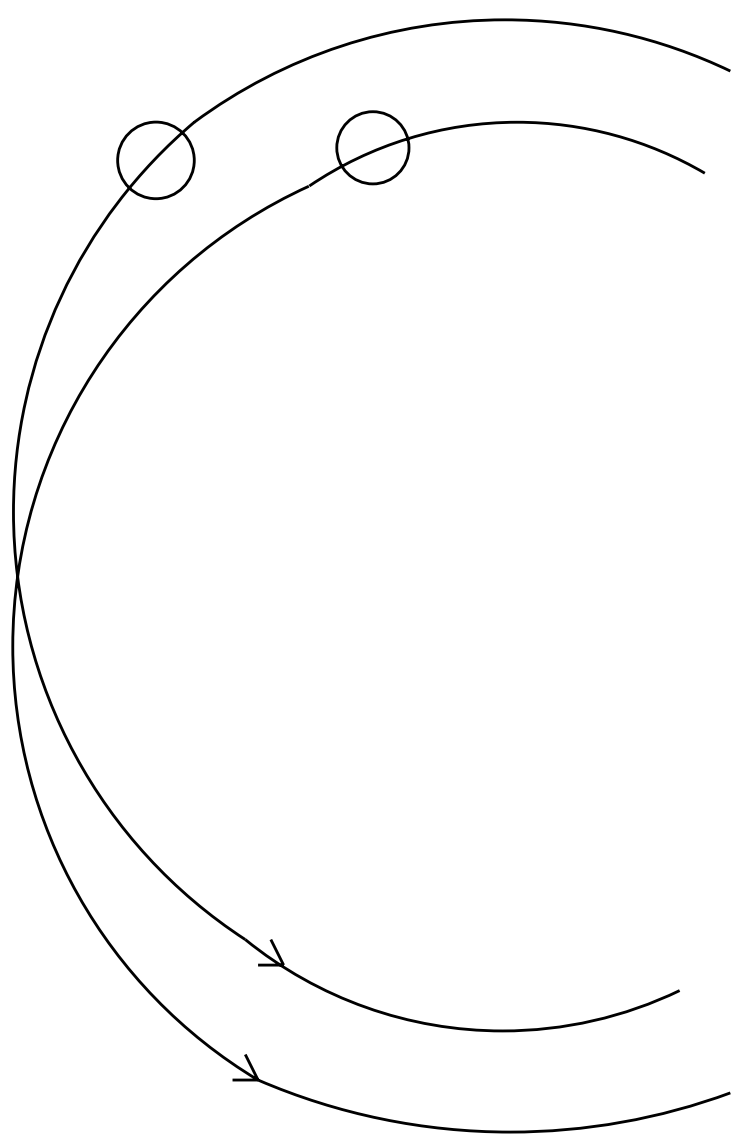

Figure 1.1. Schematic of coorbital satellites exchanging orbits. The actual exchange takes several revolutions.

motion. This technique was successfully used by Spirig and Waldvogel [13]. A second approach is that of Murray and Dermott [9]. They consider perturbations of the tangential component of the forces caused by the interactions of the two moons. Their work gives very accurate predictions of the orbits.

In the classical literature, the study of the dynamics of the three body problem, in which two of the bodies are small compared to the third, has a long and noble history. Periodic orbits of this problem in which the interaction between the two small bodies is treated as a perturbation of decoupled Kepler problems have been studied extensively (see, for example, 


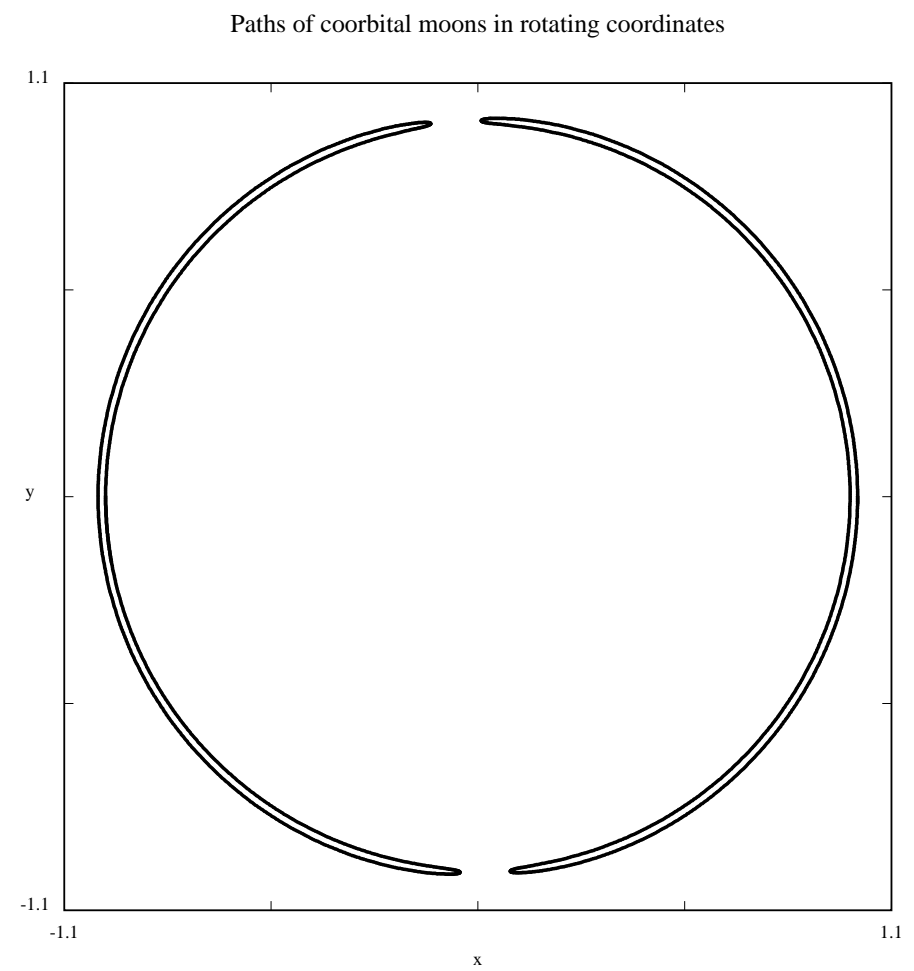

Figure 1.2. Typical coorbital paths in rotating coordinates. The angle of closest approach is the angle of the gap between the paths of the moons. In this picture, the initial positions are $(1.01,0),(0,-0.99)$; the initial velocities are $(0.0995037),(0,-1.00504)$; and the masses are $0.01^{2.4}$. (Computed with dstool.)

Meyer [6], [7]). The situation we study here involves two small masses in nearly circular coplanar orbits about a large mass. Periodic orbits of this type were described by Poincaré as periodic orbits of the "first type" (see Poincaré [11, pp. 75-76] and Goroff's introduction in [11, p. I46]) The problem studied here differs from the classical problem in that we consider solutions for which the distance between the radii of the orbits of the small bodies tends to zero as their masses tend to zero. For this reason, there is no "restricted" (small masses $=0$ ) limit, and we must study the problem for small but nonzero masses.

There are three small quantities to consider. The first is the ratio of the difference between the radii of the moons' orbits and the average radius of their orbits. We call this quantity $\epsilon$. The second is the masses of the small moons. We call the masses $\mu_{i} \epsilon^{a}$, where $\mu_{1}, \mu_{2}$ are order one. Finally, we are seeking orbits for which the moons do not pass through syzygy on the same side of the planet. Hence, there is some minimum angle between the rays from the planet to the moons. This minimum angular separation of the moons is the third small quantity; we call it $\epsilon^{b}$. (This angle is the gap between the orbits of the moons in rotating coordinates; see Figure 1.2.) The main and new result of this paper is the determination of the relative size of these small quantities that admits coorbital motion like that observed for Janus and Epimetheus. In particular, we show the following. 
Theorem 1.1. Let $\epsilon$ be the ratio of the difference between the radii of the orbits of the small moons with the average radius, and suppose the ratio of the moons' masses to the mass of the planet is of order $\epsilon^{a}$ and the minimum angular separation of the moons is of order $\epsilon^{b}$. If $2<a<5 / 2$, then the system admits coorbital motion, and in this case $b=a-2$.

The technique we use is to make restrictions on the parameters $a$ and $b$ so as to obtain a low order system in $\epsilon$ that we can analyze. Then we show coorbital motion occurs in this truncated system. Restrictions on the parameter $a$ correspond to restricting the choice of masses of the small bodies. Restrictions on the parameter $b$ correspond to limiting our attention to certain regions of the phase space. Hence we can treat $a$ and $b$ as independent parameters. Once we fix the ranges for $a$ and $b$, we must (a postiori) show that coorbital motion occurs for the masses and in the region of phase space satisfying the restrictions.

The range of values for the parameter $a$ is chosen so that the terms giving the force between the moons have only one summand of low order in epsilon. Care must be taken because terms involving epsilon appear in both the numerators and denominators of terms in the Hamiltonian.

In the final section, we discuss qualitative aspects of coorbital motion. We do not prove stability, but we discuss a lower bound for the persistence of these orbits.

2. Equations of motion and scalings. In this section, we derive our model for the study of motion of small coorbital moons. Since the two small moons have masses of the same order of magnitude, we require that they be treated equally by the equations of motion.

We begin with the Hamiltonian system representing the Newtonian three body problem given by the usual energy function

$$
H(q, p)=\frac{p_{0}^{2}}{2 M}+\frac{p_{1}^{2}}{2 m_{1}}+\frac{p_{2}^{2}}{2 m_{3}}-\frac{G M m_{1}}{\left\|q_{1}-q_{0}\right\|}-\frac{G M m_{2}}{\left\|q_{2}-q_{0}\right\|}-\frac{G m_{1} m_{2}}{\left\|q_{2}-q_{1}\right\|}
$$

where $q_{0}, p_{0}$ are the positions and momentum in the plane of the large planet with mass $M$, $q_{i}, p_{i}, i=1,2$, are the position and momenta in the plane of the small moons with masses $m_{i}, i=1,2$, respectively, and $G$ is the gravitational constant. We choose units of distance so that $G=1$ (see Meyer [8]) and units of mass so that $M=1$. This gives the Hamiltonian

$$
H(q, p)=\frac{p_{0}^{2}}{2}+\frac{p_{1}^{2}}{2 m_{1}}+\frac{p_{2}^{2}}{2 m_{2}}-\frac{m_{1}}{\left\|q_{1}-q_{0}\right\|}-\frac{m_{2}}{\left\|q_{2}-q_{0}\right\|}-\frac{m_{1} m_{2}}{\left\|q_{2}-q_{1}\right\|}
$$

Next we isolate the total momentum and center of mass via a change of variables. The usual coordinate choice for this step is the Jacobi coordinates (see Meyer [8]); however, these coordinates treat the moons asymmetrically. We use a coordinate change which treats the moons equivalently. We define new position variables $u$ by

$$
\begin{aligned}
& u_{1}=q_{1}-q_{0}, \\
& u_{2}=q_{2}-q_{0}, \\
& u_{3}=\frac{1}{1+m_{1}+m_{2}}\left(q_{0}+m_{1} q_{1}+m_{2} q_{2}\right) .
\end{aligned}
$$


Extending this to a symplectic transformation, we define new momentum variables $v$ by

$$
\begin{aligned}
& v_{1}=-\frac{m_{1}}{1+m_{1}+m_{2}} p_{0}+\frac{1+m_{2}}{1+m_{1}+m_{2}} p_{1}-\frac{m_{1}}{1+m_{1}+m_{2}} p_{2}, \\
& v_{2}=-\frac{m_{2}}{1+m_{1}+m_{2}} p_{0}-\frac{m_{2}}{1+m_{1}+m_{2}} p_{1}+\frac{1+m_{1}}{1+m_{1}+m_{2}} p_{2}, \\
& v_{3}=p_{0}+p_{1}+p_{2} .
\end{aligned}
$$

In these variables, the new Hamiltonian function is

$$
H(u, v)=\frac{\left(1+m_{1}\right) v_{1}^{2}}{2 m_{1}}+\frac{\left(1+m_{2}\right) v_{2}^{2}}{2 m_{2}}+v_{1} v_{2}+\frac{v_{3}^{2}}{2\left(1+m_{1}+m_{2}\right)}-\frac{m_{1}}{\left\|u_{1}\right\|}-\frac{m_{2}}{\left\|u_{2}\right\|}-\frac{m_{1} m_{2}}{\left\|u_{1}-u_{2}\right\|} .
$$

Since the variables $v_{3}, u_{3}$ represent the total momentum and center of mass, respectively, it suffices to consider only $u_{1}, u_{2}, v_{1}, v_{2}$.

2.1. The small quantities: Moon masses. As stated above, we take advantage of the fact that the coorbital motion involves three small quantities. We assume that the distance between the semimajor axes of the orbits of the small moons when they are far apart is of order epsilon compared to the average of the two semimajor axes. We assume that the masses are (both) of order $\epsilon^{a}$ (in comparison to the mass of the planet $M=1$ ) and that the angle of closest approach of the bodies is of order $\epsilon^{b}$.

It is convenient to incorporate the small masses first. We let

$$
\begin{aligned}
& m_{1}=\epsilon^{a} \mu_{1}, \\
& m_{2}=\epsilon^{a} \mu_{2},
\end{aligned}
$$

where $\mu_{1}, \mu_{2}$ are of the same order as the mass of the planet $M=1$. We simultaneously rescale the momentum variables, defining new momentum variables $w_{1}, w_{2}$ by

$$
\begin{aligned}
& w_{1}=\epsilon^{-a} v_{1}, \\
& w_{2}=\epsilon^{-a} v_{2} .
\end{aligned}
$$

The change from $u, v$ to $u, w$ variables is a symplectic transformation with multiplier $\epsilon^{-a}$ (see Meyer [8]). The resulting system has Hamiltonian function

$$
H(u, w)=\frac{w_{1}^{2}}{2 \mu_{1}}+\epsilon^{a} \frac{w_{1}^{2}}{2}+\frac{w_{2}^{2}}{2 \mu_{2}}+\epsilon^{a} \frac{w_{2}^{2}}{2}+\epsilon^{a} w_{1} w_{2}-\frac{\mu_{1}}{\left\|u_{1}\right\|}-\frac{\mu_{2}}{\left\|u_{2}\right\|}-\frac{\epsilon^{a} \mu_{1} \mu_{2}}{\left\|u_{1}-u_{2}\right\|},
$$

and the corresponding differential equations are

$$
\begin{aligned}
\dot{u_{1}} & =\frac{w_{1}}{\mu_{1}}+\epsilon^{a} w_{1}+\epsilon^{a} w_{2}, \\
\dot{u_{2}} & =\frac{w_{2}}{\mu_{2}}+\epsilon^{a} w_{2}+\epsilon^{a} w_{1}, \\
\dot{w_{1}} & =-\frac{\mu_{1}}{\left\|u_{1}\right\|^{3}} u_{1}+\frac{\epsilon^{a} \mu_{1} \mu_{2}}{\left\|u_{2}-u_{1}\right\|^{3}}\left(u_{2}-u_{1}\right), \\
\dot{w_{2}} & =-\frac{\mu_{2}}{\left\|u_{2}\right\|^{3}} u_{2}+\frac{\epsilon^{a} \mu_{1} \mu_{2}}{\left\|u_{2}-u_{1}\right\|^{3}}\left(u_{1}-u_{2}\right) .
\end{aligned}
$$


2.2. Incorporating the minimum distance between moons. The next step is to group terms in the system above with respect to powers of $\epsilon$. Our goal is to collect terms so that the system corresponding to the lowest order is simple yet reflects the dynamics of coorbital motion. In particular, we cannot just treat all terms involving epsilon as "higher order" since the resulting system is just two decoupled Kepler problems.

To begin, we make the a priori assumption that $a>0$, that is, that the small masses are small in comparison to the mass of the primary $M=1$. In addition, since we are seeking coorbital motion, we assume that the moons do not approach the planet, i.e., that $\left\|u_{1}\right\|$ and $\left\|u_{2}\right\|$ are of order one. Finally, while coorbital motion is subtle, it probably does not include times when the velocity is very large. Hence we assume that the (scaled) momentum variables $w_{1}, w_{2}$ are of order one. These a priori assumptions restrict our attention to a region of phase space in which we hope to find solutions corresponding to coorbital motion.

With the a priori assumptions above, we can classify all of the terms with a factor $\epsilon^{a}$ in the Hamiltonian and/or the differential equations as order $O\left(\epsilon^{a}\right)$ except the terms having

$$
\left\|u_{2}-u_{1}\right\|
$$

in the denominator. Since we are looking for orbits where the two moons do interact significantly, we must assume that the distance between the moons becomes sufficiently small that the interaction terms are significant. In particular, we assume that

$$
\left\|u_{2}-u_{1}\right\| \geq O\left(\epsilon^{b}\right)
$$

and that the minimum value of $\left\|u_{2}-u_{1}\right\|$ along an orbit is of this order. This can be interpreted as allowing the algebra to do the division between the "inner" and the "outer" solutions in the matching method. We set the relative size of $a$ and $b$ below to achieve this division.

Hence, at this stage, we have

$$
H(u, w)=\frac{w_{1}^{2}}{2 \mu_{1}}+\frac{w_{2}^{2}}{2 \mu_{2}}-\frac{\mu_{1}}{\left\|u_{1}\right\|}-\frac{\mu_{2}}{\left\|u_{2}\right\|}-\frac{\epsilon^{a} \mu_{1} \mu_{2}}{\left\|u_{2}-u_{1}\right\|}+O\left(\epsilon^{a}\right) .
$$

2.3. Rotating polar coordinates. We next change to rotating coordinates in the usual way. Let

$$
\begin{aligned}
& x_{j}=R(t) u_{j}, \\
& y_{j}=R(t) w_{j},
\end{aligned}
$$

where

$$
R(t)=\left[\begin{array}{cc}
\cos t & \sin t \\
-\sin t & \cos t
\end{array}\right]
$$

We focus on orbits of period near $2 \pi$ and hence radius near 1 without loss of generality. The new Hamiltonian is

$$
\begin{aligned}
H\left(x_{1}, x_{2}, y_{1}, y_{2}\right)= & \frac{\left\|y_{1}\right\|^{2}}{2 \mu_{1}}+\frac{\left\|y_{2}\right\|^{2}}{2 \mu_{2}}-x_{1}^{\mathrm{T}} K y_{1}-x_{2}^{\mathrm{T}} K y_{2} \\
& -\frac{\mu_{1}}{\left\|x_{1}\right\|}-\frac{\mu_{2}}{\left\|x_{2}\right\|}-\epsilon^{a} \frac{\mu_{1} \mu_{2}}{\left\|x_{2}-x_{1}\right\|}+O\left(\epsilon^{a}\right),
\end{aligned}
$$


where

$$
K=\left[\begin{array}{cc}
0 & 1 \\
-1 & 0
\end{array}\right]
$$

As expected, the next step is to convert to polar coordinates

$$
\left(r_{1}, r_{2}, \theta_{1}, \theta_{2}, R_{1}, R_{2}, \Theta_{1}, \Theta_{2}\right)=(r, \theta, R, \Theta) .
$$

In these coordinates, the Hamiltonian is given by

$$
\begin{aligned}
H(r, \theta, R, \Theta)= & \frac{1}{2 \mu_{1}}\left(R_{1}^{2}+\left(\frac{\Theta_{1}}{r_{1}}\right)^{2}\right)+\frac{1}{2 \mu_{2}}\left(R_{2}^{2}+\left(\frac{\Theta_{2}}{r_{2}}\right)^{2}\right) \\
& -\Theta_{1}-\Theta_{2}-\frac{\mu_{1}}{r_{1}}-\frac{\mu_{2}}{r_{2}} \\
& -\epsilon^{a} \frac{\mu_{1} \mu_{2}}{\left(r_{1}^{2}+r_{2}^{2}-2 r_{1} r_{2} \cos \left(\theta_{2}-\theta_{1}\right)\right)^{1 / 2}}+O\left(\epsilon^{a}\right) .
\end{aligned}
$$

2.4. Incorporating the difference between the semimajor axes. The last of the small quantities in the problem is the difference between the semimajor axes of the moons when they are far from each other and on approximate Kepler orbits. We assume this difference is of order $\epsilon$; i.e., we let

$$
\begin{aligned}
\epsilon \rho_{1} & =r_{1}-1, \\
\epsilon \rho_{2} & =r_{2}-1, \\
\epsilon \Phi_{1} & =\Theta_{1}-\mu_{1}, \\
\epsilon \Phi_{2} & =\Theta_{2}-\mu_{2} .
\end{aligned}
$$

This change of variables is a symplectic transformation with multiplier $\epsilon^{-1}$. To avoid the singularity at $\epsilon=0$, we rescale time using

$$
d t_{\text {old }} / d t_{\text {new }}=\epsilon, \text { so } t_{\text {old }}=\epsilon t_{\text {new }} .
$$

The new Hamiltonian is

$$
\begin{aligned}
& H\left(\rho_{1}, \rho_{2}, \theta_{1}, \theta_{2}, R_{1}, R_{2}, \Phi_{1}, \Phi_{2}\right)=\frac{1}{2 \mu_{1}}\left(R_{1}^{2}+\frac{\left(\mu_{1}+\epsilon \Phi_{1}\right)^{2}}{\left(1+\epsilon \rho_{1}\right)^{2}}\right)+\frac{1}{2 \mu_{2}}\left(R_{2}^{2}+\frac{\left(\mu_{2}+\epsilon \Phi_{2}\right)^{2}}{\left(1+\epsilon \rho_{2}\right)^{2}}\right) \\
& -\left(\mu_{1}+\mu_{2}\right)-\epsilon \Phi_{1}-\epsilon \Phi_{2}-\frac{\mu_{1}}{1+\epsilon \rho_{1}}-\frac{\mu_{2}}{1+\epsilon \rho_{2}} \\
& -\frac{\epsilon^{a} \mu_{1} \mu_{2}}{\left(\left(1+\epsilon \rho_{1}\right)^{2}+\left(1+\epsilon \rho_{2}\right)^{2}-2\left(1+\epsilon \rho_{1}\right)\left(1+\epsilon \rho_{2}\right) \cos \left(\theta_{2}-\theta_{1}\right)\right)^{1 / 2}}+O\left(\epsilon^{a}\right) .
\end{aligned}
$$

Again, we expand and collect similar power terms in $\epsilon$ for all but the last summand (which is treated separately below). We obtain

$$
H\left(\rho_{1}, \rho_{2}, \theta_{1}, \theta_{2}, R_{1}, R_{2}, \Phi_{1}, \Phi_{2}\right)=\frac{R_{1}^{2}}{2 \mu_{1}}+\epsilon^{2}\left(\frac{\Phi_{1}^{2}}{2 \mu_{1}}-2 \Phi_{1} \rho_{1}+\frac{\mu_{1} \rho_{1}^{2}}{2}\right)
$$




$$
\begin{gathered}
+\frac{R_{2}^{2}}{2 \mu_{2}}+\epsilon^{2}\left(\frac{\Phi_{2}^{2}}{2 \mu_{2}}-2 \Phi_{2} \rho_{2}+\frac{\mu_{2} \rho_{2}^{2}}{2}\right) \\
-\frac{\epsilon^{a} \mu_{1} \mu_{2}}{\left(\left(1+\epsilon \rho_{1}\right)^{2}+\left(1+\epsilon \rho_{2}\right)^{2}-2\left(1+\epsilon \rho_{1}\right)\left(1+\epsilon \rho_{2}\right) \cos \left(\theta_{2}-\theta_{1}\right)\right)^{1 / 2}}+O\left(\epsilon^{a}\right)+O\left(\epsilon^{3}\right),
\end{gathered}
$$

where we have kept terms of order two in $\epsilon$ and the last summand. The constant terms have no effect on the dynamics and have been dropped. The "O( $\left.\epsilon^{3}\right)$ " refers to those generated by expanding all but the last summand.

2.5. A priori assumption on the masses: Lower bound for parameter $a$. We can think of the Hamiltonian above as coming from two decoupled Kepler problems for the motion of the moons plus interaction terms. In the rotating coordinates, there is a circle of fixed points corresponding to the circular orbits at radius one. To include the fact that the moons are not on this orbit but rather are moving on orbits nearby (within $\pm \epsilon$ ), we must include the $\epsilon^{2}$ order terms in this part of the Hamiltonian.

We are seeking systems for which the terms governing interaction between the two moons are significant only when they are close. Hence we must assume that the terms collected above in "O $\left(\epsilon^{a}\right)$ " are actually of higher order than $\epsilon^{2}$. To do this, we are forced to assume that

$$
a>2 .
$$

This assumption allows the algebra to perform the localization of the interaction terms between the moons.

2.6. Interaction terms between the moons. Finally, we return to the terms governing the interaction between the two moons. We must go carefully since we must keep terms which may become large when the two moons are close.

To deal with the coupling term between the moons, we analyze

$$
g\left(\rho_{1}, \rho_{2}, \theta, \epsilon\right)=\frac{1}{\left(\left(1+\epsilon \rho_{1}\right)^{2}+\left(1+\epsilon \rho_{2}\right)^{2}-2\left(1+\epsilon \rho_{1}\right)\left(1+\epsilon \rho_{2}\right) \cos (\theta)\right)^{1 / 2}} .
$$

Here $\theta=\theta_{2}-\theta_{1}$, the angular distance between the moons. We must examine the order in epsilon of terms in the partials of $g$, particularly when $\theta$ is small. We extend our a priori assumptions to restrict our attention to orbits for which syzygy does not occur with the two moons on the same side of the planet, i.e., that

$$
\theta, 2 \pi-\theta \geq O\left(\epsilon^{b}\right)
$$

where $b$ is fixed below. We compute $\partial g / \partial \rho_{1}$ and arrange the terms as follows:

$$
\begin{aligned}
\frac{\partial g}{\partial \rho_{1}}= & -\epsilon \frac{(1-\cos \theta)+\epsilon\left(\rho_{1}-\rho_{2} \cos \theta\right)}{\left((2-2 \cos \theta)\left(1+\epsilon\left(\rho_{1}+\rho_{2}\right)+\epsilon^{2} \rho_{1} \rho_{2}\right)+\epsilon^{2}\left(\rho_{1}-\rho_{2}\right)^{2}\right)^{3 / 2}} \\
= & -\epsilon \frac{1}{2(2-2 \cos \theta)^{1 / 2}}\left[\frac{1}{\left(1+\epsilon\left(\rho_{1}+\rho_{2}\right)+\epsilon^{2} \rho_{1} \rho_{2}+\epsilon^{2}\left(\rho_{1}+\rho_{2}\right)^{2} /(2-2 \cos \theta)\right)^{3 / 2}}\right. \\
& \left.+\frac{\epsilon\left(\rho_{1}-\rho_{2} \cos \theta\right) /(1-\cos \theta)}{\left(1+\epsilon\left(\rho_{1}+\rho_{2}\right)+\epsilon^{2} \rho_{1} \rho_{2}+\epsilon^{2}\left(\rho_{1}+\rho_{2}\right)^{2} /(2-2 \cos \theta)\right)^{3 / 2}}\right] .
\end{aligned}
$$


Now, the order in epsilon of these terms is determined by the denominators. We can expand the terms within the square brackets by expanding

$$
(1+\delta)^{-3 / 2}
$$

in powers of $\delta$, where

$$
\delta=\epsilon\left(\rho_{1}+\rho_{2}\right)+\epsilon^{2} \rho_{1} \rho_{2}+\frac{\epsilon^{2}\left(\rho_{1}+\rho_{2}\right)^{2}}{(2-2 \cos \theta)} .
$$

The last summand of $\delta$ is of order $\epsilon^{2-2 b}$, so $\delta^{n}$ contains terms of order

$$
\epsilon^{k}\left(\epsilon^{2}\right)^{r}\left(\epsilon^{(2-2 b)}\right)^{n-(k+r)}=\epsilon^{k+2 r+(2-2 b)(n-k-r)} .
$$

At extreme values of $\theta$,

$$
\frac{\epsilon}{2(2-2 \cos \theta)^{1 / 2}}=O\left(\epsilon^{1-b}\right)
$$

So, expanding $\partial g / \partial \rho_{1}$ at the extreme values of $\theta$, we obtain terms of order

$$
O\left(\epsilon^{1-b+k+2 r+(2-2 b)(n-k-r)}\right) \text { and } O\left(\epsilon^{2-3 b+k+2 r+(2-2 b)(n-k-r)}\right),
$$

where $n=0,1,2, \ldots$ and $0 \leq k, r, k+r \leq n$. The terms in $\dot{R}_{1}$ are $\epsilon^{a}$ times these.

The terms in $\partial g / \partial \rho_{2}$ (which relates to the $\dot{R}_{2}=-\partial H / \partial \rho_{2}$ term) are of the same order.

For partials with respect to the $\theta$, we must compute more and be considerably more careful. First, we compute $\partial g / \partial \theta$. We could deal with the resulting terms as we did above, but it is more illustrative to examine the power series of $\partial g / \partial \theta$ in epsilon:

$$
\begin{aligned}
\frac{\partial g}{\partial \theta} & =\frac{\left(1+\epsilon \rho_{1}\right)\left(1+\epsilon \rho_{2}\right) \sin \theta}{\left((2-2 \cos \theta)\left(1+\epsilon\left(\rho_{1}+\rho_{2}\right)+\epsilon^{2} \rho_{1} \rho_{2}\right)+\epsilon^{2}\left(\rho_{1}-\rho_{2}\right)^{2}\right)^{3 / 2}} \\
& =\frac{\sin \theta}{(2-2 \cos \theta)^{3 / 2}}-\frac{1}{2} \frac{\left(\rho_{1}+\rho_{2}\right) \sin \theta}{(2-2 \cos \theta)^{3 / 2}} \epsilon \\
& +\frac{1}{4} \frac{\left(\left(3 \rho_{1}^{2}+3 \rho_{2}^{2}+2 \rho_{1} \rho_{2}\right) \cos \theta+3 \rho_{1}^{2}+3 \rho_{2}^{2}-14 \rho_{1} \rho_{2}\right) \sin \theta}{(2-2 \cos \theta)^{5 / 2}} \epsilon^{2} \\
& +-\frac{1}{8} \frac{\left(\left(5 \rho_{1}^{3}+5 \rho_{2}^{3}+3 \rho_{1}^{2} \rho_{2}+3 \rho_{1} \rho_{2}^{2}\right) \cos \theta+13 \rho_{1}^{3}+13 \rho_{2}^{3}-21 \rho_{1}^{2} \rho_{2}-21 \rho_{1} \rho_{2}^{2}\right) \sin \theta}{(2-2 \cos \theta)^{5 / 2}} \epsilon^{3}+O\left(\epsilon^{4}\right) .
\end{aligned}
$$

We analyze each summand at extreme values of $\theta$, so $2-2 \cos \theta=O\left(\epsilon^{2 b}\right)$ and $\sin \theta=O\left(\epsilon^{b}\right)$.

Evaluating the order of these terms at the extreme values of $\theta$, we see that the first term is of order $\epsilon^{-2 b}$. The second term is of order $\epsilon^{1-2 b}$, and the third term is at the largest of order $\epsilon^{2-4 b}$.

Subsequent terms are of order

$$
O\left(\epsilon^{w-2 b+k+2 r+(2-2 b)(n-k-r)}\right),
$$

where $w=0,1,2, n=1,2,3, \ldots$, and $0 \leq k, r, k+r \leq n$. The corresponding terms in the $\dot{\Phi}_{i}$ are of order $\epsilon^{a}$ times these. 
2.7. Bounds for $b$. We saw above that we must keep terms of order $\epsilon^{2}$ and lower in order to have any chance of accurately representing the motion of the two moons when they are far apart. In the previous section, we computed the order of terms resulting from the interaction between the moons when they are close to each other. The order of these terms is as follows:

$$
\begin{aligned}
\dot{R}_{i} \quad \text { contains terms } & \epsilon^{a+1-b+k+2 r+(2-2 b)(n-k-r)} \text { and } \epsilon^{a+2-3 b+k+2 r+(2-2 b)(n-k-r),} \\
& \text { where } n=0,1, \ldots \text { and } 0 \leq k, r, \quad k+r \leq n ; \\
\dot{\Phi}_{i} \quad \text { contains terms } & \epsilon^{a-2 b}, \quad \epsilon^{a-2 b+1}, \epsilon^{a-4 b+2}, \\
& \epsilon^{a+w-2 b+k+2 r+(2-2 b)(n-k-r)}, \\
& \text { where } w=0,1,2, n=1,2,3, \ldots, 0 \leq k, r, \text { and } k+r \leq n .
\end{aligned}
$$

In order to obtain a model system in which to search for and study coorbital behavior, we choose an upper bound for $a$ and conditions on $b$ which guarantee that finitely many of these terms are of order less than or equal to 2 in epsilon. We note that the terms

$$
\epsilon^{a+1-b+(2-2 b) n} \quad \text { and } \quad \epsilon^{a-2 b+(2-2 b) n}
$$

appear in $\dot{R}_{i}$ and $\dot{\Phi}_{i}$. In order to guarantee that only finitely many of these terms are of order less than 2 in epsilon, we must assume that $2-2 b>0$. Hence we assume $b<1$. That is, we restrict our attention to the region of the phase space corresponding to positions where the angular separation is greater than or equal to $O\left(\epsilon^{b}\right)$ and $b>1$.

However, we do not want to eliminate all the terms from the interactions between the moons. To guarantee that at least one interaction term is of order less than 2 in epsilon, we assume that

$$
a-2 b<2
$$

or

$$
\frac{a-2}{2}<b
$$

The simplest nontrivial system possible would contain just the one interaction term of order $\epsilon^{a-2 b}$ in $\dot{\Phi}_{i}$ and none of the higher order terms in $\dot{\Phi}_{i}$ or $\dot{R}_{i}$. To obtain this system to order 2 in epsilon, we need to guarantee that

$$
a-2 b+1>2 \quad \text { and } \quad a-4 b+2>2 .
$$

All subsequent terms of $\dot{\Phi}_{i}$ and all terms of $\dot{R}_{i}$ are of order greater than these powers and/or of order greater than $a>2$ in epsilon. If $0<b<1 / 2$, then $a-2 b+1<a-4 b+2$, so we must assume that

$$
\begin{gathered}
a-2 b+1>2, \\
\text { i.e., } \quad b<\frac{a-1}{2} .
\end{gathered}
$$

If $1 / 2 \leq b<1$, then $a-4 b+2 \leq a-2 b+1$, so we must assume that

$$
a-4 b+2>2
$$


i.e.,

$$
b<\frac{a}{4}
$$

2.8. Summary. To summarize, we have made two sorts of restrictions. First, we have restricted our attention to a specific region of the phase space in which we hope to find coorbital motion. This includes a priori restrictions on the size of some variables (e.g., the distances from the planet to the moons is of order one) and restrictions on the parameter $b$. Second, we have made restrictions on the possible values of the parameters of the masses of the moons by placing restrictions on the parameter $a$. The motivation for these restrictions is to isolate a region in phase $x$ parameter space where the lowest order system is simple enough to study (and in which we hope to find coorbital behavior).

We collect the following assumptions:

- The distances of the moons from the planet are bounded below by an order one constant.

- The velocities of the moons are bounded above by an order one constant.

- The difference between the radii of the orbits of the moons is of order $\epsilon$ (i.e., the radii of the moons' orbits are close).

- The masses of the moons are both of order $\epsilon^{a}$ (where the mass of the planet is one), and $a>0$; i.e., the moons are small.

- The angular distance between the moons (call it $\theta$ ) satisfies

$$
\min (\theta) \geq O\left(\epsilon^{b}\right)
$$

and

$$
\max (2 \pi-\theta) \geq O\left(\epsilon^{b}\right),
$$

and $b>0$ (i.e., the moons get close to each other, but syzygy of the moons does not occur with both on the same side of the planet), and at closest approach $\theta=O\left(\epsilon^{b}\right)$.

Recall that the radii of the approximate Kepler orbits of the two moons when they are separated are assumed to differ by order epsilon. In the resulting system, we must keep terms of order two in epsilon in order to distinguish our system from one where the two moons are both on the same circular orbit.

- We assume the masses of the moons are such that $a>2$. This assumption is equivalent to assuming that the significant interaction between the moons occurs only when they are close.

- We assume that the minimum angular separation of the moons is such that

$$
\frac{a-2}{2}<b
$$

This assumption ensures that there is at least one term of order less than two in epsilon resulting from the interaction between the moons.

- We assume that $b<1$. This assumption is necessary to keep the number of interaction terms between the moons of order less than two in epsilon finite.

- The two assumptions above imply that $a<4$. 
- If $0<b<1 / 2$, we assume that $b<(a-1) / 2$. If $1 / 2 \leq b<1$, we assume that $b<a / 4$. These assumptions yield the simplest nontrivial system in which to search for coorbital behavior.

With these assumptions, we can write the truncated equations (up to order $\epsilon^{2}$ ) as

$$
\begin{aligned}
\dot{\rho}_{1} & =\frac{1}{\mu_{1}} R_{1} \\
\dot{\theta}_{1} & =\epsilon^{2}\left(\frac{\Phi_{1}}{\mu_{1}}-2 \rho_{1}\right) \\
\dot{R}_{1} & =\epsilon^{2}\left(2 \Phi_{1}-\mu_{1} \rho_{1}\right), \\
\dot{\Phi}_{1} & =\epsilon^{a} \frac{\mu_{1} \mu_{2} \sin \left(\theta_{2}-\theta_{1}\right)}{\left(2-2 \cos \left(\theta_{2}-\theta_{1}\right)\right)^{3 / 2}}, \\
\dot{\rho}_{2} & =\frac{1}{\mu_{2}} R_{2} \\
\dot{\theta}_{2} & =\epsilon^{2}\left(\frac{\Phi_{2}}{\mu_{2}}-2 \rho_{2}\right) \\
\dot{R}_{2} & =\epsilon^{2}\left(2 \Phi_{2}-\mu_{2} \rho_{2}\right), \\
\dot{\Phi}_{2} & =-\epsilon^{a} \frac{\mu_{1} \mu_{2} \sin \left(\theta_{2}-\theta_{1}\right)}{\left(2-2 \cos \left(\theta_{2}-\theta_{1}\right)\right)^{3 / 2}} .
\end{aligned}
$$

This system is Hamiltonian with

$$
\begin{aligned}
H\left(\rho_{1}, \rho_{2}, \theta_{1}, \theta_{2}, R_{1}, R_{2}, \Phi_{1}, \Phi_{2}\right)= & \frac{1}{2 \mu_{1}} R_{1}^{2}+\epsilon^{2}\left(\frac{\Phi_{1}^{2}}{2 \mu_{1}}-2 \rho_{1} \Phi_{1}+\frac{\mu_{1} \rho_{1}^{2}}{2}\right) \\
& +\frac{1}{2 \mu_{1}} R_{2}^{2}+\epsilon^{2}\left(\frac{\Phi_{2}^{2}}{2 \mu_{2}}-2 \rho_{2} \Phi_{2}+\frac{\mu_{2} \rho_{2}^{2}}{2}\right) \\
& -\epsilon^{a} \frac{\mu_{1} \mu_{2}}{\left(2-2 \cos \left(\theta_{2}-\theta_{1}\right)\right)^{1 / 2}} .
\end{aligned}
$$

3. Analysis of the reduced system. Two steps remain in the construction of our reduced system. First, we must determine appropriate value(s) for the parameter $b$, which determines the minimum angular separation between the moons. Second, we must justify (a postiori) our model by finding solutions of our model exhibiting coorbital behavior.

3.1. Projecting to two degrees of freedom. The four degree of freedom system above almost decouples into two systems. We can take advantage of this, projecting the system above onto a two degree of freedom system as follows: Let

$$
\begin{aligned}
\rho & =\rho_{2}-\rho_{1}, \\
\theta & =\theta_{2}-\theta_{1}, \\
R & =R_{2} / \mu_{2}-R_{1} / \mu_{1}, \\
\Phi & =\Phi_{2} / \mu_{2}-\Phi_{1} / \mu_{1}
\end{aligned}
$$


to obtain the system

$$
\begin{aligned}
\dot{\rho} & =R, \\
\dot{\theta} & =\epsilon^{2}(\Phi-2 \rho), \\
\dot{R} & =\epsilon^{2}(2 \Phi-\rho), \\
\dot{\Phi} & =-\frac{\left(\mu_{1}+\mu_{2}\right) \epsilon^{a} \sin \theta}{(2-2 \cos \theta)^{3 / 2}} .
\end{aligned}
$$

This is a Hamiltonian system with Hamiltonian

$$
H(\rho, \theta, R, \Phi)=\frac{R^{2}}{2}+\epsilon^{2} \frac{\rho^{2}}{2}+\epsilon^{2}\left(\frac{\Phi^{2}}{2}-2 \rho \Phi\right)-\frac{\left(\mu_{1}+\mu_{2}\right) \epsilon^{a}}{(2-2 \cos \theta)^{1 / 2}}
$$

3.2. Separating fast and slow variables. Next we isolate the slow and fast variables and put the system into a simpler form while maintaining the Hamiltonian structure. Hence all of our changes of variables will be symplectic or symplectic transformations with multipliers.

First, we redistribute the $\epsilon$ on the $\rho, R$ variables by letting

$$
\begin{aligned}
& \sqrt{\epsilon} P=R, \\
& \frac{1}{\sqrt{\epsilon}} \gamma=\rho .
\end{aligned}
$$

We also rescale time, choosing a new time variable with $d t_{\text {new }} / d t_{\text {old }}=\epsilon$. Note that this change undoes the scaling done at the beginning of section 2.4, so this time variable is "real" time. These changes yield the new system

$$
\begin{aligned}
\dot{\gamma} & =P \\
\dot{\theta} & =-2 \epsilon^{1 / 2} \gamma+\epsilon \Phi, \\
\dot{P} & =-\gamma+2 \epsilon^{1 / 2} \Phi \\
\dot{\Phi} & =-\frac{\left(\mu_{1}+\mu_{2}\right) \epsilon^{a-1} \sin \theta}{(2-2 \cos \theta)^{3 / 2}}
\end{aligned}
$$

The Hamiltonian is

$$
H(\gamma, \theta, P, \Phi)=\frac{P^{2}}{2}+\frac{\gamma^{2}}{2}-2 \epsilon^{1 / 2} \Phi \gamma+\epsilon \frac{\Phi^{2}}{2}+\epsilon^{a-1} \frac{\mu_{1}+\mu_{2}}{(2-2 \cos \theta)^{1 / 2}} .
$$

To lowest order, the $\gamma, P$ equations are those of a harmonic oscillator with equilibrium at $P=0, \gamma=2 \epsilon^{1 / 2} \Phi$. We move this equilibrium to the origin by changing variables, setting

$$
\tilde{\gamma}=\gamma-2 \epsilon^{1 / 2} \Phi
$$

However, to keep the change symplectic, we must also alter the other variables. Using the generating function (see Meyer [8])

$$
S(\gamma, \theta, \tilde{P}, \tilde{\Phi})=\gamma \tilde{P}+\theta \tilde{\Phi}-2 \epsilon^{1 / 2} \tilde{\Phi} \tilde{P}
$$


we obtain the symplectic change of variables

$$
\begin{aligned}
\tilde{\gamma} & =\gamma-2 \epsilon^{1 / 2} \tilde{\Phi}, \\
\tilde{\theta} & =\theta-2 \epsilon^{1 / 2} \tilde{P}, \\
\tilde{P} & =P, \\
\tilde{\Phi} & =\Phi .
\end{aligned}
$$

In these variables, the Hamiltonian is

$$
H(\tilde{\gamma}, \tilde{\theta}, \tilde{P}, \tilde{\Phi})=\frac{\tilde{P}^{2}}{2}+\frac{\tilde{\gamma}^{2}}{2}-\epsilon \frac{3}{2} \tilde{\Phi}^{2}-\epsilon^{a-1} \frac{\mu_{1}+\mu_{2}}{\left(2-2 \cos \left(\tilde{\theta}+2 \epsilon^{1 / 2} \tilde{P}\right)\right)^{1 / 2}}
$$

As above, we expand and keep only terms which contribute terms of order less than or equal to one in epsilon to the differential equation. We must identify these terms in the expansions of

$$
\frac{\partial}{\partial \tilde{\theta}}\left(\epsilon^{a-1} \frac{\mu_{1}+\mu_{2}}{\left(2-2 \cos \left(\tilde{\theta}+\epsilon^{1 / 2} \tilde{P}\right)\right)^{1 / 2}}\right)
$$

and

$$
\frac{\partial}{\partial \tilde{P}}\left(\epsilon^{a-1} \frac{\mu_{1}+\mu_{2}}{\left(2-2 \cos \left(\tilde{\theta}+\epsilon^{1 / 2} \tilde{P}\right)\right)^{1 / 2}}\right) .
$$

The computations involved are similar to those of sections 2.6 and 2.7,

$$
\begin{aligned}
& \frac{\partial}{\partial \tilde{\theta}}\left(\frac{1}{\left(2-2 \cos \left(\tilde{\theta}+\epsilon^{1 / 2} \tilde{P}\right)\right)^{1 / 2}}\right)=-\frac{\sin \tilde{\theta}}{(2-2 \cos \tilde{\theta})^{3 / 2}} \\
& +\epsilon^{1 / 2} \tilde{P}\left(-\frac{\cos \tilde{\theta}}{(2-2 \cos \tilde{\theta})^{3 / 2}}+\frac{\sin ^{2} \tilde{\theta}}{(2-2 \cos \tilde{\theta})^{5 / 2}}\right)+\cdots,
\end{aligned}
$$

where "..." refers to terms in higher powers of $\epsilon^{1 / 2} \tilde{P}$. As above, we examine the higher order terms at the extreme values of $\tilde{\theta}$. In addition, we use the fact that restricting our attention to the region of the phase space where $\rho$ is of order one implies that $\gamma$ is $O\left(\epsilon^{1 / 2}\right)$. Since $\gamma$ and $P$ behave to lowest order like a harmonic oscillator about $(\epsilon \Phi, 0)$ and $\Phi$ is order one, we restrict our attention to the region of phase space where $\tilde{P}$ is $O\left(\epsilon^{1 / 2}\right)$. Assuming $\gamma$ and $P$ are order $O\left(\epsilon^{1 / 2}\right)$ is equivalent to assuming that the eccentricity of the moons' orbits is small $(O(\epsilon))$ when they are far apart.

However, we also need to add the assumption that

$$
\frac{a-1}{3}>b \text {. }
$$

Using this and $0<b<1$, we have that we need only keep the first summand on the right-hand side above in the equation for $\dot{\Phi}$.

Similarly, we need to assume that

$$
\frac{2 a-3}{4}>b
$$


to guarantee that all terms of

$$
\frac{\partial}{\partial \tilde{P}}\left(\epsilon^{a-1} \frac{\mu_{1}+\mu_{2}}{\left(2-2 \cos \left(\tilde{\theta}+\epsilon^{1 / 2} \tilde{P}\right)\right)^{1 / 2}}\right)
$$

are of order greater than epsilon in the equation for $\dot{\rho}$.

Hence, to first order in epsilon, we have

$$
H(\gamma, \theta, P, \Phi)=\frac{P^{2}}{2}+\frac{\gamma^{2}}{2}-\epsilon \frac{3}{2} \Phi^{2}-\epsilon^{a-1} \frac{\mu_{1}+\mu_{2}}{(2-2 \cos (\theta))^{1 / 2}} .
$$

We emphasize that this is the reduced system under the assumptions and restrictions of section 2.8 and that

- $P$ is $O\left(\epsilon^{1 / 2}\right)$,

- $b<(a-1) / 3$, and $b<(2 a-3) / 4$.

3.3. Fixing the range for $a$ and setting $b$. We note that in these variables, to lowest order in $\epsilon$, we have the system

$$
\begin{aligned}
\dot{\gamma} & =P, \\
\dot{\theta} & =-3 \epsilon \Phi, \\
\dot{P} & =-\gamma, \\
\dot{\Phi} & =-\frac{\left(\mu_{1}+\mu_{2}\right) \epsilon^{a-1} \sin \theta}{(2-2 \cos \theta)^{3 / 2}} .
\end{aligned}
$$

This system decouples. The Hamiltonian for the $\theta, \Phi$ system is

$$
K(\theta, \Phi)=-\frac{3}{2} \epsilon \Phi^{2}-\frac{\left(\mu_{1}+\mu_{2}\right) \epsilon^{a-1}}{(2-2 \cos \theta)^{1 / 2}} .
$$

Using this expression, we see that solutions of the slow system are closed curves about $\theta=\pi, \Phi=0$. These correspond to the coorbital motions of the two moons. The angle $\theta$, which equals the angle between the moons, oscillates strictly between 0 and $2 \pi$, while the angular velocity $\Phi$ alternates sign depending on which moon is closer to the planet. The oscillations of $\gamma$ and $P$ correspond to slight eccentricities of the Kepler orbits followed by the moons when they are far apart.

Hence we have found the coorbital solutions in the truncated system, and this provides a postiori justification of the assumptions necessary to obtain the system.

Moreover, if we consider the level set $K(\theta, \Phi)=k$, then on $\theta=\pi, \Phi=\Phi_{0}$ we have

$$
K\left(\pi, \Phi_{0}\right)=k=-\epsilon \frac{3}{2} \Phi_{0}^{2}-\epsilon^{a-1} \frac{\mu_{1}+\mu_{2}}{2} \approx-\epsilon \frac{3}{2} \Phi_{0}^{2} .
$$

The maximum, $\theta_{\max }$, and minimum, $\theta_{\min }$, values of $\theta$ on the level curve $K(\theta, \Phi)=k$ occur where $\Phi=0$, and so, to lowest order in $\epsilon$,

$$
\theta_{\min } \approx \epsilon^{a-2} \frac{2\left(\mu_{1}+\mu_{2}\right)}{3 \Phi_{0}^{2}}
$$


and similarly for $\theta_{\max }$.

This implies that the consistent choice for the parameter $b$ is $b=a-2$. Combining this with the a priori assumptions of section 2.8, we obtain the following: if $0<b<1 / 2$, then $2<a<5 / 2$, while if $1 / 2 \leq b<1$, then $2<a<8 / 3$. Adding the assumptions of section 3.2 , namely, that $b<(a-1) / 3$ and $b<(2 a-3) / 4$, and using $a>2, b=a-2$, we obtain $2<a<5 / 2$.

This completes the proof of the theorem stated in the introduction.

3.4. Analysis of the moons of Saturn. We next consider examples of coorbital motion observed in the moons of Saturn - first Janus and Epimetheus. The analysis above allows us to relate the three small quantities in coorbital motion, the difference in average radius of the orbit, the mass, and the angle of closest approach. The equation at the end of section 3.3 relates $\theta_{\min }, \mu_{1}, \mu_{2}$, and $\Phi_{0}$ the value of $\Phi$ when $\theta=\pi$ to each other. Referring back to the system of section 3.1, we see that if we take the initial value of $\rho$ (the separation of the radii) to be one, then nearly circular orbits will have $\rho \approx 2 \Phi$, so we take $\Phi_{0}=1 / 2$. Substituting gives

$$
\theta_{\min } \approx \frac{8}{3} \epsilon^{a-2}\left(\mu_{1}+\mu_{2}\right)=\frac{8}{3} \epsilon^{-2}\left(m_{1}+m_{2}\right),
$$

where we use that the masses satisfy $m_{i}=\epsilon^{a} \mu_{i}$. This agrees to lowest order with the similar relationship found by Murray and Dermott [9].

While careful observation has accurately determined the orbits of Janus and Epimetheus (see Nicholson et al. [10]), the masses cannot be directly measured. In practice, masses of small bodies are determined by the effect these bodies have on other bodies. For Janus and Epimetheus, observation yields that the average radius of the orbits of the moons is 150,432 $\mathrm{Km}$ and the difference in the radii when they are far apart is approximately $50 \mathrm{Km}$. This gives an epsilon value of $\epsilon \approx 3.32 \cdot 10^{-4}$. Consistent choices of masses and minimum separation angles which agree with observation are given by $m_{1}+m_{2} \approx 4.5 \cdot 10^{-9}$ and $\theta_{\min } \approx 6^{\circ}$. While our theorem specifies only the order of magnitude of the relationship between the masses and epsilon, the value $4.5 \cdot 10^{-9} \approx \epsilon^{2.4}$ is well within the range allowed, i.e., $\epsilon^{2} \approx 1 \cdot 10^{-7}$ to $\epsilon^{2.5} \approx 2 \cdot 10^{-9}$.

Other groups of moons of Saturn have very close semimajor axes - for example, Dione and Helene and Thethys, Telesto, and Calypso (see Murray and Dermott [9]). However, the ratios of the masses of the smaller moon to the larger one in these cases is so small that it is more natural to treat these as perturbations of the restricted three body problem. One exception is the pair Prometheus and Pandora, which have approximately equal masses and radii 139,350 $\mathrm{Km}$ and 141,700 Km, respectively (data from Murray and Dermott [9, pp. 531-533]). For this pair, the average radius is $140,525 \mathrm{Km}$ and

$$
\epsilon=\frac{141,700-139,350}{140,525} \approx 0.0167 \text {. }
$$

The minimum mass ratio of these moons with Saturn necessary for them to exhibit coorbital motion is on the order of $0.0167^{5 / 2}=3.6 \cdot 10^{-5}$. The actual mass ratio is $2.37 \cdot 10^{-10}$ and, as expected, these moons do not switch orbits. The $\mathrm{F}$ ring is between the orbits of these two moons. However, the interaction terms between these moons have a significant effect on their orbits as recent observations and simulations show (see Goldreich and Rapporport [3]). 
4. Persistence of coorbital motion. Above we found, under appropriate assumptions on the parameters, orbits in a certain region of the phase space of the three body problem for a truncated approximation of the full system. Ideally we would like to show that there is a large set of such orbits which remains in the region for all time ("stability" of coorbital motion). We can, however, use ideas from KAM theory to at least discuss a lower bound on the minimum time solution's stay in the coorbital region. Here we assume the small moons are of equal mass $\left(\mu_{1}=\mu_{2}=1\right)$.

In section 3.1, we gave a projection of the full four degree of freedom system onto a two degree of freedom system. In the case in which $\mu_{1}=\mu_{2}=1$, this projection can easily be altered and extended to form a symplectic transformation as follows:

$$
\begin{aligned}
\rho & =\rho_{2}-\rho_{1}, & \bar{\rho} & =\rho_{2}+\rho_{1}, \\
\theta & =\theta_{2}-\theta_{1}, & \bar{\theta} & =\theta_{2}+\theta_{1}, \\
R & =\left(R_{2}-R_{1}\right) / 2, & \bar{R} & =\left(R_{2}+R_{1}\right) / 2, \\
\Phi & =\left(\Phi_{2}-\Phi_{1}\right) / 2, & \bar{\Phi} & =\left(\Phi_{2}+\Phi_{1}\right) / 2 .
\end{aligned}
$$

The new Hamiltonian is

$$
\begin{aligned}
H(\rho, \bar{\rho}, \theta, \bar{\theta}, R, \bar{R}, \Phi, \bar{\Phi})= & R^{2}+\bar{R}^{2}+\frac{\epsilon^{2}}{4}\left(\rho^{2}+\bar{\rho}^{2}\right)+\epsilon^{2}\left(\Phi^{2}+\bar{\Phi}^{2}-\rho \Phi-\bar{\rho} \bar{\Phi}\right) \\
& -\epsilon^{a} \frac{1}{(2-2 \cos (\theta))^{1 / 2}} .
\end{aligned}
$$

As in section 3.2, we separate the fast and slow variables by setting

$$
\begin{array}{ll}
\sqrt{\epsilon} P=R, & \sqrt{\epsilon} \bar{P}=\bar{R}, \\
\frac{1}{\sqrt{\epsilon}} \gamma=\rho, & \frac{1}{\sqrt{\epsilon}} \bar{\gamma}=\bar{\rho}
\end{array}
$$

and rescale time to obtain

$$
\begin{aligned}
H(\gamma, \bar{\gamma}, \theta, \bar{\theta}, P, \bar{P}, \Phi, \bar{\Phi})= & P^{2}+\bar{P}^{2}+\frac{1}{4}\left(\gamma^{2}+\bar{\gamma}^{2}\right)+\epsilon^{1 / 2} \gamma \Phi-\epsilon^{1 / 2} \bar{\gamma} \bar{\Phi}+\epsilon \Phi^{2}+\epsilon \bar{\Phi}^{2} \\
& -\epsilon^{a-1} \frac{1}{(2-2 \cos (\theta))^{1 / 2}}
\end{aligned}
$$

Finally, we let

$$
\begin{aligned}
\tilde{\gamma} & =\gamma-2 \epsilon^{1 / 2} \Phi, & \tilde{\bar{\gamma}} & =\bar{\gamma}-2 \epsilon^{1 / 2} \bar{\Phi} \\
\tilde{\theta} & =\theta-2 \epsilon^{1 / 2} P, & \tilde{\bar{\theta}} & =\bar{\theta}-2 \epsilon^{1 / 2} \bar{P} .
\end{aligned}
$$

To reduce excessive decoration, we remove the tildes, obtaining the Hamiltonian to first order in $\epsilon$ :

$$
\begin{aligned}
H(\gamma, \bar{\gamma}, \theta, \bar{\theta}, P, \bar{P}, \Phi, \bar{\Phi})= & P^{2}+\bar{P}^{2}+\frac{1}{4}\left(\gamma^{2}+\bar{\gamma}^{2}\right)+2 \epsilon \Phi^{2}+2 \epsilon \bar{\Phi}^{2} \\
& -\epsilon^{a-1} \frac{1}{(2-2 \cos (\theta))^{1 / 2}}
\end{aligned}
$$

We note that $\bar{\theta}$ is missing from the Hamiltonian. Hence

$$
\dot{\bar{\Phi}}=0 \text {. }
$$


This corresponds to the conservation of angular momentum. The conjugate variable $\bar{\theta}$ satisfies

$$
\dot{\bar{\theta}}=4 \epsilon \bar{\Phi}
$$

which corresponds to the constant rotation of the angle at which the closest approach occurs. (The outer moon takes slightly longer to traverse $360^{\circ}$ than the inner moon, so the position of closest approach precesses.) We can remove these variables from consideration.

Also, we note that, to order one in epsilon, the unbarred and barred systems decouple. In fact, while there could be terms of order between one and $a-1$ in epsilon which we have not displayed above, the two systems actually decouple up to order $a-1$ in epsilon. (The changes of variables in section 3 do not mix barred with unbarred variables.)

4.1. Period of the slow system and a twist map. We can compute the approximate period of the slow unbarred system as follows: Letting $T$ equal the period of the orbit containing the point $\theta=\pi, \Phi=\Phi_{0}$, we can use the symmetry of the system across the $\theta$ axis and the monotonicity of $\theta$ when $\Phi$ has constant sign to say that

$$
T=2 \int_{\theta_{\min }}^{\theta_{\max }} \frac{d t}{d \theta} d \theta .
$$

Substituting in the differential equation for $\dot{\theta}$, we obtain

$$
T=2 \int_{\theta_{\min }}^{\theta_{\max }} \frac{1}{3 \epsilon \Phi} d \theta,
$$

where the relationship between $\theta$ and $\Phi$ is given by $K(\theta, \Phi)$ in section 3.3.

For the orbit with initial conditions $\theta=\pi, \Phi=\Phi_{0}$, we have $1 / \Phi \approx 1 / \Phi_{0}$ on most of the interval $\left(\theta_{\min }, \theta_{\max }\right)$. Also, the values of $\theta_{\min }$ and $\theta_{\max }$ change at higher order in epsilon as $\Phi$ is changed, and hence the period $T$ decreases as $\Phi_{0}$ increases.

This implies that if we put the $\theta, \Phi$ and $\gamma, P$ variables into action-angle coordinates (to order one in epsilon) in the four dimensional system (decoupled from $\bar{\gamma}, \bar{P}$ ), then the return map in $\theta, \Phi$ on a constant energy surface plane given by fixing $\gamma$ and $P$ satisfies a twist condition. Because the periods in $\theta, \Phi$ and $\gamma, P$ are order epsilon apart, the resulting map will be a "small twist." Luckily, both Poincaré's last geometric theorem and the KAM theorem apply in this situation (see Franks [2] and Levy and Moser [5]), providing periodic orbits and invariant circles for the return map and their perturbations.

4.2. Persistence of coorbital motion. Unfortunately, as we noted above, the barred and unbarred systems decouple only to order $a-1$ in epsilon. Hence invariant circles for the return map of the unbarred system correspond to separating invariant sets in the constant energy manifolds of the full system truncated to order $a-1$ in epsilon. We can go slightly beyond this order by looking at the terms we studied at the beginning of section 2.7 since these are the first terms involving both barred and unbarred variables. The term of lowest order is $\epsilon^{a-2 b}=\epsilon^{4-a}$.

This implies that if we follow solutions of the full four degree of freedoms system, the value of the integral $K(\theta, \Phi)$ can change no faster than a constant times $\epsilon^{4-a}$. Hence our coorbital solutions remain qualitatively the same for a time period of order $1 / \epsilon^{4-a}$. 
For Janus and Epimetheus, one unit of time corresponds to one orbit around Saturn or 0.694590 days (see Murray and Dermott [9]). With $\epsilon=3.32 \cdot 10^{-4}$ and $a=2.4$, we conclude that the orbits of these moons will remain qualitatively the same for at least on the order of $1 / \epsilon^{1.6}$ orbits. This is on the order of 368,000 orbits or 700 years.

This ignores the possibility of large coefficients in the expansion; i.e., it cannot be taken literally - only as an order of magnitude estimate. The precise statement is only that the rate of change of $K(\theta, \Phi)$ is of order $\epsilon^{4-a}$ for orbits in the region of the phase space that we are considering.

Acknowledgments. The authors thank Tasso Kaper for his valuable suggestions and patient listening and the referees for useful suggestions.

\section{REFERENCES}

[1] K. Aksnes, The tiny satellites of Jupiter and Saturn and their interactions with the rings, in Stability of the Solar System and Its Minor Natural and Artificial Bodies, V. G. Szebehely, ed., D. Reidel, Boston, MA, 1985, pp. 3-16.

[2] J. Franks, A variation on the Poincaré-Birkhoff theorem, in Hamiltonian Dynamical Systems, Contemp. Math. 81, AMS, Providence, RI, 1988, pp. 111-117.

[3] P. Goldreich AND N. RAPPAPORT, Chaotic Motions of F-Ring Shepherds, http://www.arxiv.org/abs/astro-ph/0205330.

[4] J. Llibre AND M. OllÉ, The motion of Saturn coorbital satellites in the restricted three-body problem, Astron. Astrophys., 378 (2001), pp. 1087-1099.

[5] M. Levi AND J. Moser, A Lagrangian proof of the invariant curve theorem for twist mappings, in Smooth Ergodic Theory and Its Applications, Proc. Sympos. Pure Math. 69, A. Katok, R. de la Llave, Y. Pesin, and H. Weiss, eds., AMS, Providence, RI, 2001, pp. 733-746.

[6] K. R. Meyer, Symmetries and integrals in mechanics, in Dynamical Systems, M. M. Peixoto, ed., Academic Press, New York, 1973, pp. 259-272

[7] K. R. Meyer, Periodic Solutions of the N-Body Problem, Lecture Notes in Math. 1719, Springer-Verlag, Berlin, 1999.

[8] K. R. Meyer And G. R. Hall, Introduction to Hamiltonian Dynamical Systems and the N-Body Problem, Appl. Math. Sci. 90, Springer-Verlag, New York, 1992.

[9] C. D. Murray and S. F. Dermott, Solar System Dynamics, Cambridge University Press, Cambridge, UK, 1999.

[10] P. D. Nicholson, D. P. Hamilton, K. Matthews, and C. Yoder, New observations of Saturn's coorbital satellites, Icarus, 100 (1982), pp. 464-484.

[11] H. Poincaré, New Methods of Celestial Mechanics, D. Goroff, ed., American Institute of Physics, New York, 1993.

[12] H. Salo And C. F. Yoder, The dynamics of coorbital satellite systems, Astron. Astrophys., 205 (1988), pp. 309-327.

[13] F. SPIRIG AND J. WALdVOGEL, The three-body problem with two small masses: A singular-perturbation approach to the problem of Saturn's coorbiting satellites, in Stability of the Solar System and Its Minor Natural and Artificial Bodies, V. G. Szebehely, ed., D. Reidel, Boston, MA, 1985, pp. 223-234. 